\title{
"Peeling off" Mechanism of Asphaltenes from Solid/Liquid Interface in the Presence of Highly Charged Amphiphilic Macromolecule
}

Ting Chen, ${ }^{a b}$ Fanghui Liu, ${ }^{a}$ Shizhe Huang, ${ }^{a b}$ Wei Zhang, ${ }^{a}$ Hui Wang, ${ }^{a}$ Qingfeng Hou, ${ }^{c}$ Donghong Guo, ${ }^{c}$ Aiqing Ma, ${ }^{d}$ Keji Sun, ${ }^{d}$ Hui Yang ${ }^{* a}$ and Jinben Wang ${ }^{a}$

${ }^{\mathrm{a}}$ Key Laboratory of Colloid, Interface and Chemical Thermodynamics, Institute of Chemistry, Chinese Academy of Sciences, Beijing 100190, P. R. China

${ }^{\mathrm{b}}$ University of Chinese Academy of Sciences, Beijing 100049, P. R. China

${ }^{\mathrm{c}}$ Key Laboratory of Oilfield Chemistry, Research Institute of Petroleum Exploration and Development (RIPED), CNPC, Beijing 100083, P. R. China

dOil Production Technology Research Institute, Shengli Oilfield Branch Company, Sinopec, Dongying, Shandong 257000, P. R. China

*To whom correspondence should be addressed.

E-mail: yanghui@iccas.ac.cn (H. Yang). Tel: +8610-62523395.

Fax: 8610-62523395. 
Gel Permeation Chromatography. The polydispersity and molecular weights of PASBn were measured by GPC, as listed as following.

Table S1. GPC Results of PASBn.

\begin{tabular}{ccccc}
\hline Homopolymer & $\mathrm{M}_{\mathrm{n}}$ & $\mathrm{M}_{\mathrm{w}}$ & $\mathrm{M}_{\mathrm{w}} / \mathrm{M}_{\mathrm{n}}$ & $\mathrm{Pd}$ \\
\hline PASB $_{\mathrm{n}}$ & 6109 & 7952 & 1.30 & 1.30 \\
\hline
\end{tabular}


Elemental Analysis. The elemental composition of the asphaltenes (C, N, O, S) was determined by multi-functional X-ray photoelectron spectrometer (XPS, ESCALab250Xi, VG, England) and, C, H, and N were determined by an elemental analyzer (FLASH EA1112, Italy). The high percentage of heteroatoms in the molecules endowed the asphaltenes as highly polar property entities. Asphaltenes contained a range of functional groups, such as $-\mathrm{COOH},-\mathrm{OH},-\mathrm{NH}_{2}$, and polycyclic aromatic rings (see Table S2).

Table S2. Elemental Analysis of Asphaltene by XPS.

\begin{tabular}{cc}
\hline element type & molar percentage (\%) \\
\hline $\mathrm{C}$ & 72.15 \\
$\mathrm{~N}$ & 0.85 \\
$\mathrm{O}$ & 15.88 \\
$\mathrm{~S}$ & 1.11 \\
$\mathrm{Si}$ & 10.01 \\
\hline
\end{tabular}


Density and viscosity. Density was measured with a laboratory density meter (DMA $4500 \mathrm{M}$, Anton Paar, Austria), with a repeatability of $5 \times 10^{-5} \mathrm{~g} \cdot \mathrm{cm}^{-3}$. The principle was based on the oscillating tube method and calibration with air and Milli-Q water. A built-in Peltier thermostat maintained the measuring temperature at $25{ }^{\circ} \mathrm{C}$. Dynamic viscosity was calculated on the basis of kinematic viscosity measurements through microviscometers (Lovis $2000 \mathrm{M} / \mathrm{ME}$, Anton Paar, Austria), with an accuracy of $0.5 \%$. A flow time of Newtonian liquids in a capillary was measured. The viscometer was immersed in a thermostatic bath at $25{ }^{\circ} \mathrm{C}$. The measured values of density and viscosity were presented at Table S3.

Table S3. Density and viscosity of MilliQ water and polyelectrolyte solutions.

\begin{tabular}{cccc}
\hline & $\begin{array}{c}\text { concentration } \\
(\mathrm{g} / \mathrm{L})\end{array}$ & $\begin{array}{c}\text { viscosity } \\
(\mathrm{mpa} . \mathrm{s})\end{array}$ & $\begin{array}{c}\text { density } \\
\left(\mathrm{g} / \mathrm{cm}^{3}\right)\end{array}$ \\
\hline MilliQ water & - & 0.8937 & 0.99703 \\
\hline Toluene & - & 0.5866 & 0.866 \\
\hline \multirow{2}{*}{ PASBn } & 0.5 & 1.9358 & 0.99714 \\
& 1.0 & 2.2961 & 0.99721 \\
& 5.0 & 5.5665 & 0.99786 \\
& 10.0 & 6.7616 & 0.99911 \\
& 20.0 & 11.4302 & 1.00081 \\
\hline & 0.5 & 0.9256 & 0.99715 \\
PASC $_{1}$ & 1.0 & 0.9482 & 0.99700 \\
& 5.0 & 1.0764 & 0.99831 \\
& 10.0 & 3.0545 & 0.99980 \\
& 20.0 & 10.2201 & 1.00261 \\
\hline
\end{tabular}


Desorption process of asphaltenes exposed to PASBn at the concentration of 0.5 $\mathrm{g} / \mathrm{L}$ versus time was fitted with a double-exponential function as described:

$$
y=A_{1} \mathrm{e}^{b_{1} x}+A_{2} e^{b_{2} x}
$$

where $A_{1}$ and $A_{2}$ are the coefficient, $b_{1}$ and $b_{2}$ are the decay length. The parameters (with 95\% confidence bounds) used for a double-exponential function fitting are as follows:

Table S4. Parameters used for a double-exponential function fitting of desorption process of asphaltenes.

\section{Parameters}

\begin{tabular}{cccc}
\hline$A_{1}$ & $b_{1}$ & $A_{2}$ & $b_{2}$ \\
5843 & $-1.232 \mathrm{E}-5$ & -5869 & -5.983 \\
\hline
\end{tabular}




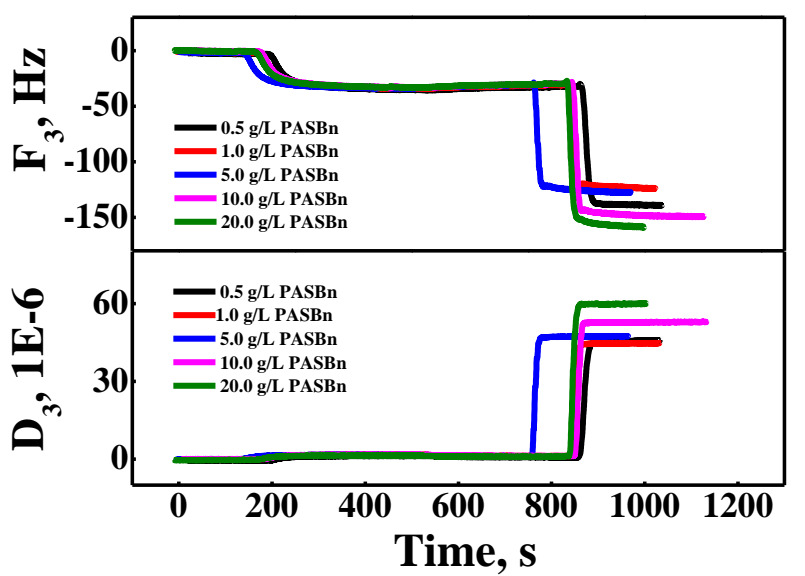

(a)

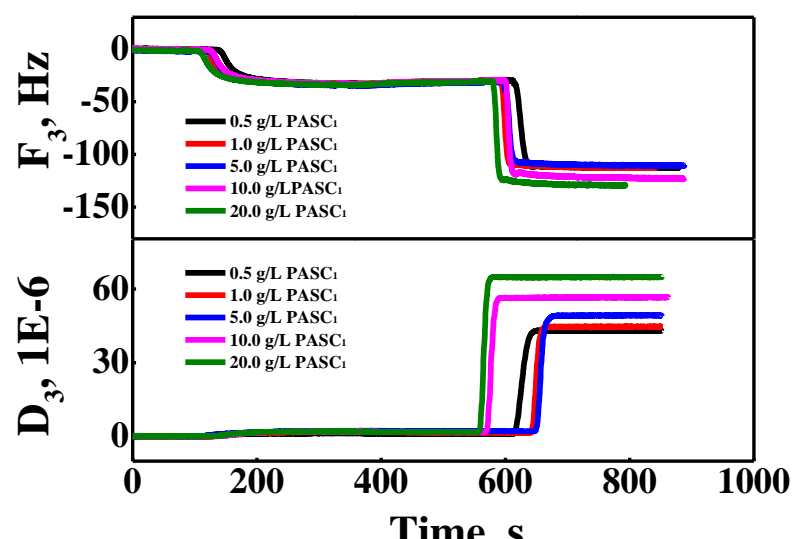

(b)

Figure S1.Changes in frequency and dissipation factor versus time at different concentrations of (a) PASBn and (b) PASC $_{1}$. 
Micropolarity. Pyrene was employed as a fluorescence probe at a concentration of 1 $\mu \mathrm{M}$ and the sample solutions were stirred at room temperature overnight before measurement. Steady-state fluorescence spectra were obtained by a Hitachi F-4500 spectrofluorometer at $25.0 \pm 0.5{ }^{\circ} \mathrm{C}$. The emission spectra were scanned from 350 to $550 \mathrm{~nm}$ using a $335 \mathrm{~nm}$ excitation wavelength. The width of the emission slit was 2.5 $\mathrm{nm}$. In emission spectra, the relative intensities of the first vibronic peak $\left(I_{1}\right)$ and the third vibronic peak $\left(I_{3}\right)$ are sensitive to the environmentpolarity, which are frequently used for the determination of aggregate polarity and critical aggregation concentration.

In the presence of PASBn, with the increase of concentration, $I_{1} / I_{3}$ values remain constant at first and then decrease markedly, revealing the formation of aggregates and the appearance of CAC of around $1.2 \mathrm{~g} / \mathrm{L}$. Different from PASBn, $I_{1} / I_{3}$ values almost reach a constant value at measured concentration of $\mathrm{PASC}_{1}$, indicating that $\mathrm{PASC}_{1}$ molecules cannot form aggregates due to the lack of polarity segments.

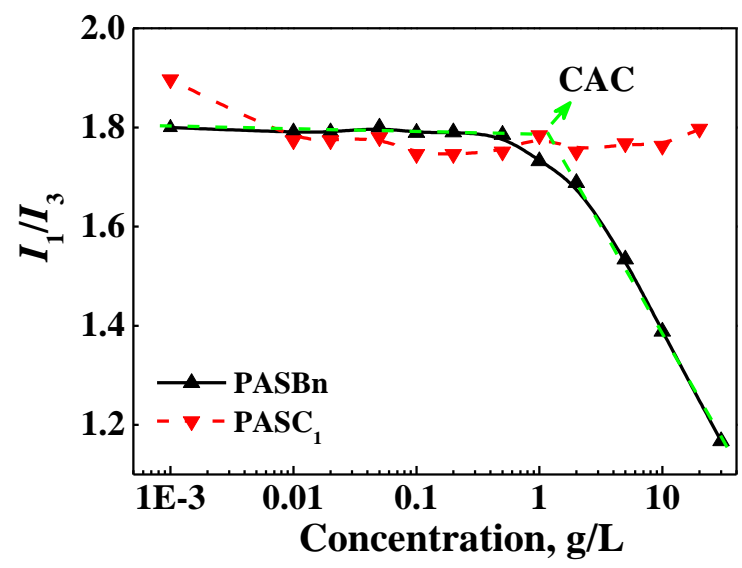

Figure S2. Plots of fluorescence intensity ratio of $I_{1} / I_{3}$ versus concentration of PASBn and $\mathrm{PASC}_{1}$. 
The partial enlarged images of asphaltene-coated film in the presence of $10.0 \mathrm{~g} / \mathrm{L}$ PASBn solutions was compared with $10.0 \mathrm{~g} / \mathrm{L}$ PASBn-coated film on bare substrate in the same dimension, as shown in Figure S3. From Figure (a) and (c), a rough and heterogeneous surface with an Root Mean Square (RMS) roughness more than 1.65 $\mathrm{nm}$ can be observed, whereas the morphology of $10.0 \mathrm{~g} / \mathrm{L}$ PASBn-coated film on bare substrate was relatively smooth and homogenous with a small RMS roughness. The "cavities" and "islands" in Figure (a) are mainly related to the asphaltene desorption or polymer aggregates adsorption. The analogous morphology of PASBn aggregates with the same scale adsorbed on the silica surface can be seen from the Figure (b) and (d), which further confirmed the competitive adsorption between asphaltenes and polyelectrolytes.
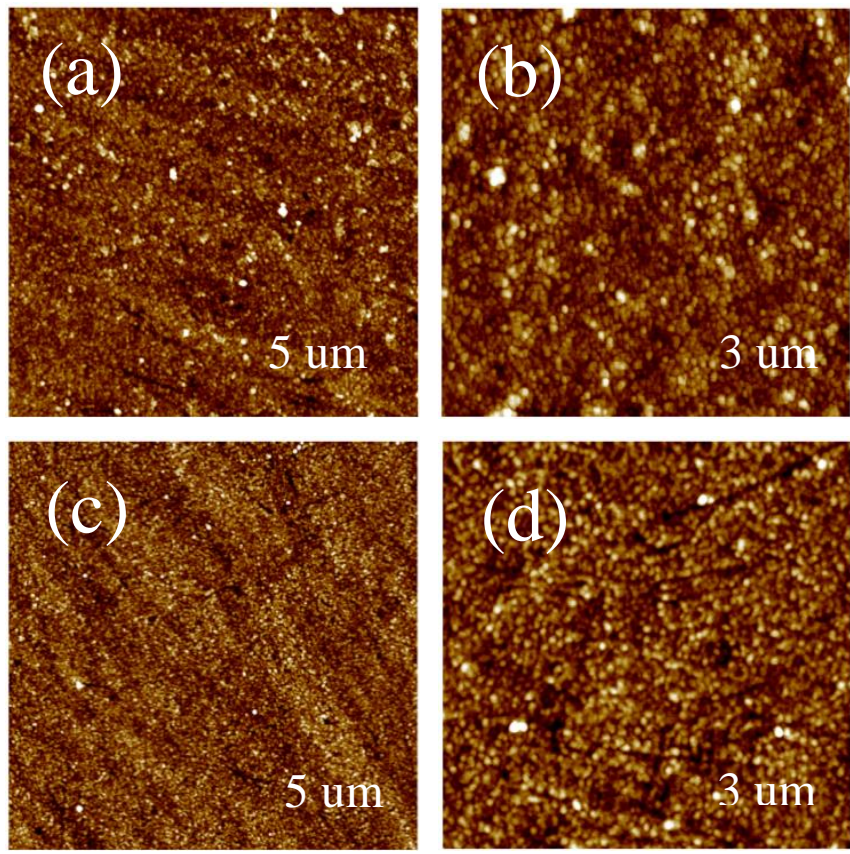

Figure S3. AFM images of the QCM-D samples: asphaltene-coated film introduced PASBn of $10.0 \mathrm{~g} / \mathrm{L}$ in (a) $5 \mu \mathrm{m} \times 5 \mu \mathrm{m}$; (b) $3 \mu \mathrm{m} \times 3 \mu \mathrm{m}$; $10.0 \mathrm{~g} / \mathrm{L}$ PASBn-coated film on bare substrate (c) $5 \mu \mathrm{m} \times 5 \mu \mathrm{m}$; (d) $3 \mu \mathrm{m} \times 3 \mu \mathrm{m}$. 


\section{References}

[1] S. Acevedo, M.a.A. Ranaudo, C. García, J. Castillo, A. Fernández, M. Caetano, S. Goncalvez. Importance of asphaltene aggregation in solution in determining the adsorption of this sample on mineral surfaces. Colloid Surf. A-Physicochem. Eng. Asp 2000; 166: 145-52.

[2] S. Wang, N. Segin, K. Wang, J.H. Masliyah, Z. Xu. Wettability control mechanism of highly contaminated hydrophilic silica/alumina surfaces by ethyl cellulose. J. Phys. Chem. C 2011; 115: 10576-87.

[3] U. Farooq, J. Sjöblom, G. Øye. Desorption of Asphaltenes from Silica-Coated Quartz Crystal Surfaces in Low Saline Aqueous Solutions. J. Dispersion Sci. Technol 2011; 32: 1388-95.

[4] M. Nourani, T. Tichelkamp, B. Gaweł, G. Øye. Method for Determining the Amount of Crude Oil Desorbed from Silica and Aluminosilica Surfaces upon Exposure to Combined Low-Salinity Water and Surfactant Solutions. Energy Fuels 2014; 28: 1884-9. 